05.3

\title{
Свободная энергия образования зародыша при росте III-V нитевидного нанокристалла
}

\author{
() В.Г. Дубровский ${ }^{1}$, А.С. Соколовский ${ }^{1}$, И.В. Штром ${ }^{2}$ \\ ${ }^{1}$ Университет ИТМО, Санкт-Петербург, Россия \\ ${ }^{2}$ Санкт-Петербургский государственный университет, Санкт-Петербург, Россия \\ E-mail: dubrovskii@mail.ioffe.ru
}

Поступило в Редакцию 28 мая 2020 г.

В окончательной редакции 28 мая 2020 г.

Принято к публикации 4 июня 2020 г.

\begin{abstract}
Получено выражение для свободной энергии образования зародыша из жидкой капли катализатора при росте III-V нитевидных нанокристаллов по механизму пар-жидкость-кристалл. Проведен учет эффекта истощения количества атомов элемента группы V (As) в капле за счет роста островка при осаждении As из газовой фазы. Теоретически исследованы различные режимы формирования островка, в том числе режим с остановкой роста при малых концентрациях As в капле. Показано, что остановка роста происходит при уменьшении концентрации Аs до равновесного значения. Полученные результаты могут быть использованы при моделировании кинетики роста III-V нитевидных нанокристаллов, статистики их нуклеации и функций распределения по длине, кристаллической фазы и процессов легирования.
\end{abstract}

Ключевые слова: III-V нитевидный нанокристалл, зародыш, свободная энергия.

DOI: 10.21883/PJTF.2020.18.49991.18401

Нитевидные нанокристаллы (ННК) полупроводниковых соединений III-V представляют интерес с фундаментальной точки зрения, а также для приложений в нанофотонике [1-4]. III-V ННК обычно выращиваются по механизму пар-жидкость-кристалл (ПЖК) [5], где в качестве катализаторов используются капли $\mathrm{Au}[5,6]$ или металла группы III [7]. Одним из преимуществ ННК перед другими наноструктурами, например квантовыми точками [8], является эффективная релаксация упругих напряжений на боковой поверхности. Это позволяет выращивать III-V ННК на кремнии, а также гетероструктуры в ННК высокой степени кристаллического совершенства $[9,10]$.

Важную роль при исследовании кинетики формирования III-V ННК, морфологии их ростового интерфейса и кристаллической фазы (кубическая или вюрцитная) играет in situ диагностика роста внутри просвечивающего электронного микроскопа [11-13]. В работах $[12,13]$ было продемонстрировано, что при автокаталитическом росте вюрцитных ННК GaAs из капель Ga рост двумерного зародыша GaAs происходит в два этапа. На первом этапе островки растут очень быстро, практически мгновенно, а после достижения некоторого „размера остановки“ начинается второй, медленный этап роста со скоростью поступления атомов As из пара. Данный эффект связан с истощением концентрации As в капле на первом этапе роста, что характерно для нуклеации в наносистемах [14-16]. В работе [17] дано выражение для производной работы образования по числу частиц, определяющей критический размер и размер остановки, в предположении фиксированного числа атомов As в капле. Целью настоящей работы являются вывод и анализ выражения для самой свободной энергии образо- вания зародыша в присутствии материального контакта капли с паром, что необходимо при моделировании кинетики роста III-V ННК [16-18], их функций распределения по длине, кристаллической фазы и процессов легирования $[19,20]$.

Рассмотрим систему, состоящую из капли жидкого раствора Ga и As (ограничиваемся случаем автокаталитического ПЖК-роста), в которую поступают атомы $\mathrm{Ga}$ и As из газовой фазы, и двумерного островка GaAs. Исследуем изменение свободной энергии системы в процессе, представленном на рис. 1. В начальном состоянии имеем $l_{0}$ атомов As в капле и $l_{t o t}-l_{0}$ атомов $\mathrm{Ga}$ в капле, где $l_{\text {tot }}$ - количество всех атомов в капле, $g$ атомов $\mathrm{Ga}$ и $g$ атомов As в газовой фазе или паре (с химическими потенциалами $\mu_{3}^{v}$ и $\mu_{5}^{v}$ в тепловых единицах $\left.k_{\mathrm{B}} T\right)$. Химические потенциалы атомов $\mathrm{Ga}\left(\mu_{3}\right)$ и As $\left(\mu_{5}\right)$ в жидкости в начальном состоянии зависят от удельной атомной концентрации As $c_{5}=l_{0} / l_{t o t}$ и абсолютной температуры $T$. В финальном состоянии имеем $l_{0}-i+j$ атомов As в капле, $l_{t o t}-\left(l_{0}-i+j\right)$ атомов $\mathrm{Ga}$ в капле, $i$ из которых перешло в островок, состоящий из $i$ пар GaAs (с химическим потенциалом $\mu_{35}$ ), и $j$ пар GaAs поступило в каплю из газовой фазы. Соответственно число атомов Ga и As в газе стало равным $g-j$. Химические потенциалы атомов Ga и As после образования островка зависят от финальной концентрации As, которая стала равной $c_{5}^{\prime}=\left(l_{0}-i+j\right) /\left(l_{t o t}-2 i+2 j\right)$, где $l_{\text {tot }}-2 i+2 j$ есть число всех атомов в капле в финальном состоянии. Изменение объемной части свободной энергии системы (в единицах $k_{\mathrm{B}} T$ ) в таком процессе равно

$$
\begin{aligned}
F_{V}= & -j \Delta \mu_{V S}-(i-j) \Delta \mu_{L S}\left(c_{5}^{\prime}\right)+l_{0}\left[\mu_{5}\left(c_{5}^{\prime}\right)-\mu_{5}\left(c_{5}\right)\right] \\
& +\left(l_{t o t}-l_{0}\right)\left[\mu_{3}\left(c_{5}^{\prime}\right)-\mu_{3}\left(c_{5}\right)\right] .
\end{aligned}
$$




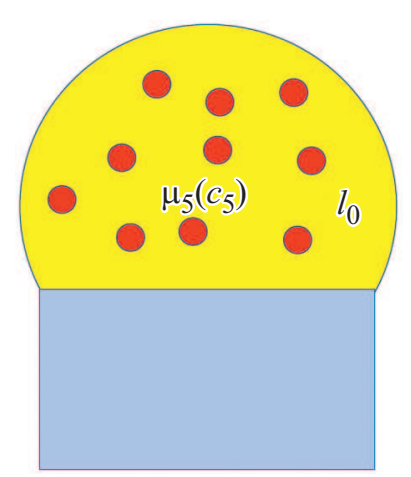

Initial state

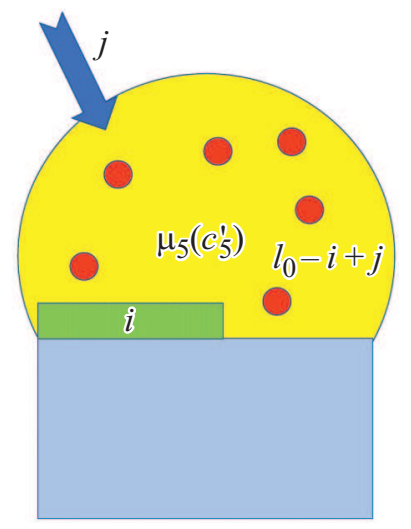

Final state
Рис. 1. Начальное состояние системы из капли с $l_{0}$ атомами As в капле и III-V ННК без островка и ее конечное состояние с островком из $i$ пар GaAs и капли с оставшимися $l_{0}-i+j$ атомами As, $j$ из которых поступили из пара и $i$ перешли в островок.

Здесь $\Delta \mu_{V S}=\mu_{3}^{v}+\mu_{5}^{v}-\mu_{35}$ есть разность химических потенциалов на пару $\mathrm{GaAs}$ в паре и кристалле, $\Delta \mu_{L S}\left(c_{5}^{\prime}\right)=\mu_{3}\left(c_{5}^{\prime}\right)+\mu_{5}\left(c_{5}^{\prime}\right)-\mu_{35}-$ разность химических потенциалов на пару GaAs в жидкости и кристалле, зависящая от финальной концентрации As $c_{5}^{\prime}$. В случае автокаталитического ПЖК-роста всегда справедливо $c_{3}=1-c_{5}$ и $c_{3}^{\prime}=1-c_{5}^{\prime}$.

В соответствии с соотношением Гиббса-Дюгема для бинарной системы имеем

$$
d \mu_{3}=-\frac{c_{5}^{\prime}}{c_{3}^{\prime}} d \mu_{5}
$$

Учтем теперь, что в связи с высокой летучестью As его концентрация в капле всегда много меньше концентрации $\mathrm{Ga}: c_{5} \ll c_{3}[16-20]$. Тогда $c_{3}^{\prime}=1-c_{5}^{\prime} \cong 1$ и $\mu_{5}\left(c_{5}^{\prime}\right) \cong \mu_{5}^{P}+\ln c_{5}^{\prime}$, где $\mu_{5}^{P}-$ химический потенциал чистой жидкости, состоящей из атомов As. Используя эти приближения и интегрируя выражение (2), получаем $\mu_{3}\left(c_{5}^{\prime}\right)-\mu_{3}\left(c_{5}\right)=c_{5}-c_{5}^{\prime}$. В том же приближении можно считать $l_{\text {tot }}-2(i-j) \cong l_{\text {tot }}$, поэтому

$$
\left(l_{t o t}-l_{0}\right)\left[\mu_{3}\left(c_{5}^{\prime}\right)-\mu_{3}\left(c_{5}\right)\right]=i-j .
$$

Для идеального раствора As в жидком Ga справедливо

$$
l_{0}\left[\mu_{5}\left(c_{5}^{\prime}\right)-\mu_{5}\left(c_{5}\right)\right]=l_{0} \ln \left(\frac{c_{5}^{\prime}}{c_{5}}\right)=l_{0} \ln \left(\frac{l_{0}-i+j}{l_{0}}\right) .
$$

Поскольку $\mu_{3}\left(c_{5}^{\prime}\right) \cong \mu_{3}^{P}, \quad$ мы можем представить $\Delta \mu_{L S}\left(c_{5}^{\prime}\right)$ в виде

$$
\begin{aligned}
\Delta \mu_{L S}\left(c_{5}^{\prime}\right) & \cong \mu_{3}^{P}+\mu_{5}^{P}-\mu_{35}+\ln c_{5}^{\prime} \\
& =\ln \left(\frac{c_{5}^{\prime}}{c_{5}^{e q}}\right)=\ln \left(\frac{l_{0}-i+j}{l_{e q}}\right) .
\end{aligned}
$$

В приведенных выражениях $\mu_{3}^{P}-$ химический потенциал жидкого $\mathrm{Ga}, c_{5}^{e q}-$ равновесная концентрация As, $l_{e q}-$ соответствующее ей число атомов As в капле.
Используя выражения (3), (4) и (5) в (2), получаем

$$
\begin{aligned}
F_{V}= & -j \Delta \mu_{V S}-(i-j) \ln \left(\frac{l_{0}-i+j}{l_{e q}}\right) \\
& +l_{0} \ln \left(\frac{l_{0}-i+j}{l_{0}}\right)+i-j .
\end{aligned}
$$

В случае мгновенного роста $(j=0)$ полученное выражение сводится к

$$
F_{V, 0}=-i \ln \left(\frac{l_{0}-i}{l_{e q}}\right)+l_{0} \ln \left(\frac{l_{0}-i}{l_{0}}\right)+i .
$$

Легко проверить, что

$$
\frac{\partial F_{V}}{\partial i}=-\ln \left(\frac{l_{0}-i+j}{l_{e q}}\right) .
$$

При $j=0$ это совпадает с результатом работы [17].

Полная свободная энергия образования зародыша $F$ равна сумме объемной энергии $F_{V}$ и поверхностной энергии боковой границы зародыша $F_{S}$. Аппроксимация поверхностной энергии островка, справедливая при любом размере, была предложена Гласом в работах $[12,17]$. Ee удобно представить в виде функции степени заполнения островком монослоя ННК $\theta=i / i_{\mathrm{ML}}$, где $i_{\mathrm{ML}}=(3 \sqrt{3} / 2) R^{2} h / \Omega_{35}$ - число пар GaAs в монослое ННК, $R$ - радиус ННК в виде правильного шестиугольника с радиусом $R$, измеряемым от центра к вершине шестиугольника, $h$ - высота монослоя, $\Omega_{35}$ - элементарный объем на пару GaAs в ННК. Вводя степени заполнения $\theta_{0}=l_{0} / i_{\mathrm{ML}}$ и $\theta_{e q}=l_{e q} / i_{\mathrm{ML}}$, а также заполнение $x=j / i_{\mathrm{ML}}$, соответствующее накачке из газовой фазы, и используя формулу Гласа для поверхностной энергии с полиномами первой степени [17], получаем, что окончательный результат для свободной энергии образования зародыша имеет вид

$$
\begin{aligned}
f= & \frac{F}{i_{\mathrm{ML}}}=-x \Delta \mu_{V S}-(\theta-x) \ln \left(\frac{\theta_{0}-\theta+x}{\theta_{e q}}\right) \\
& +\theta_{0} \ln \left(1-\frac{\theta-x}{\theta_{0}}\right)+\theta-x+\alpha\left[A \theta^{1 / 2}\right. \\
& \left.+B(1-\theta)^{1 / 2}-B+(1-A+B) \theta\right] .
\end{aligned}
$$

Здесь $\alpha=4 \gamma_{0} \Omega_{35} / \sqrt{3} k_{\mathrm{B}} T R$ - параметр, пропорциональный эффективной поверхностной энергии боковой грани ННК $\gamma_{0}[12]$, а коэффициенты равны $A=3.3748$ и $B=2.2993$ [17].

Качественное исследование выражения (9) приводит к следующим выводам. Процесс роста монослоя ННК начинается при $\theta=0$ (в момент нуклеации зародыша) и заканчивается при $\theta=1$, когда островок заполняет всю верхнюю грань ННК. Полный цикл формирования монослоя заканчивается при $x=1$, когда количество материала, содержащееся в монослое ННК, добавлено в каплю из пара. Свободная энергия формирования 


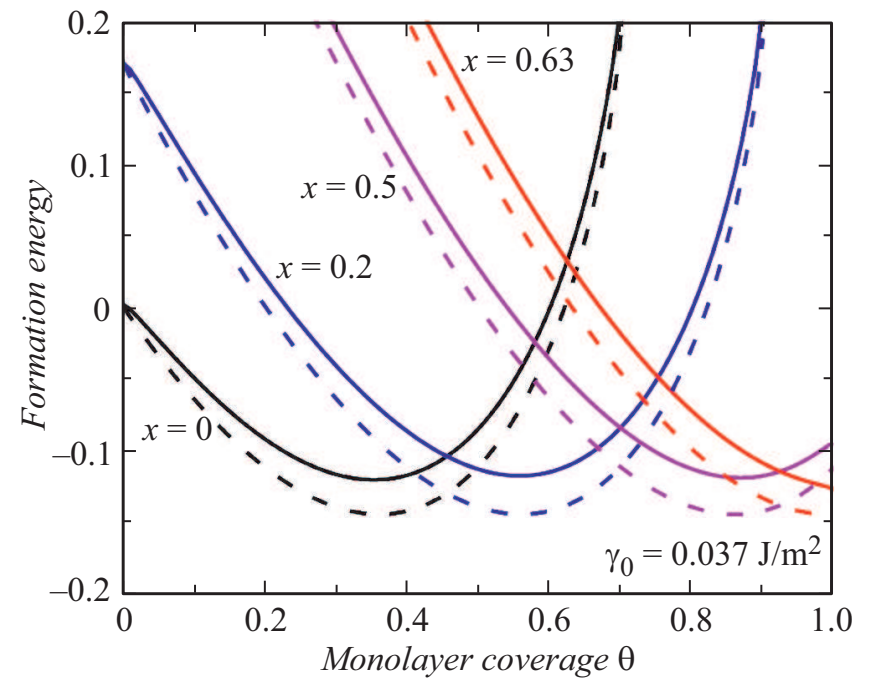

Рис. 2. Приведенная свободная энергия образования зародыша монослоя НHК GaAs $f+x \Delta \mu_{V S}$ как функция степени заполнения $\theta$ при различных значениях $x$, указанных около кривых. Графики получены на основе выражений (9),(10) при радиусе ННК $R=20 \mathrm{~nm}$, температуре $T=610^{\circ} \mathrm{C}, \theta_{e q}=0.34$, $\theta_{0}=0.7$ и низком значении поверхностной энергии боковой грани ННК $\gamma_{0}=0.037 \mathrm{~J} / \mathrm{m}^{2}$, соответствующем $\alpha=0.01584$. Штриховые линии соответствуют объемной части свободной энергии без учета вклада от поверхности зародыша и дают идентичные значения минимума для размера островка во всех случаях.

полного монослоя ННК равна $f(1)=-\Delta \mu_{V S}+\alpha$, что соответствует переходу $i_{\mathrm{ML}}$ пар GaAs из газовой фазы в кристалл, формированию боковой поверхности ННК монослойной высоты и полному восстановлению степени пересыщения в капле.

Поскольку $\partial F / \partial i=\partial f / \partial \theta$, размер остановки $\theta_{s}[16]$, по достижении которого прекращается быстрый рост островка из жидкости, определяется из решения уравнения $\partial f / \partial \theta=0$, соответствующего минимуму $f(\theta)$ (максимум в области малых заполнений соответствует критическому размеру при нуклеации зародыша). Экстремумы $f(\theta)$ находятся из уравнения

$$
-\ln \left(\frac{\theta_{0}-\theta+x}{\theta_{e q}}\right)+\alpha \frac{d \Gamma}{d \theta}=0,
$$

где $\Gamma(\theta)$ - безразмерная поверхностная энергия в квадратных скобках выражения (9). При малых $\alpha \ll 1$, соответствующих достаточно большим радиусам ННК $R$ и малым поверхностным энергиям $\gamma_{0}$, влияние поверхности на размер остановки пренебрежимо мало $[18,19]$. В этом случае размер остановки равен $\theta_{s}=\theta_{0}-\theta_{e q}+x[18,19]$, что переходит в $\theta_{s}=\theta_{0}-\theta_{e q}$ при мгновенном росте. Очевидно, что размер остановки соответствует падению концентрации As в капле до равновесного значения.

Согласно теории нуклеации [20], скорость роста островка в свободномолекулярном режиме обмена атомами As между жидкостью и кристаллом равна $d i / d t=[1-\exp (\partial F / \partial i)] / \tau$, где $l-$ число атомов As, находящихся в данный момент времени в составе капли, $\tau$ - характерное время роста. Пренебрегая поверхностной энергией, получаем $\partial F / \partial i \cong \partial F_{V} / \partial i$. Используя формулу (8) и замечая, что $l_{0}-i+j=l$, получаем закон роста островка в виде $d i / d t=\left(l-l_{e q}\right) / \tau$, что в терминах заполнений совпадает с [17-19]:

$$
\frac{d \theta}{d t}=\frac{\theta_{L}-\theta_{e q}}{\tau}
$$

где $\theta_{L}=l / i_{\mathrm{ML}}-$ есть эквивалентное заполнение жидкости. В отсутствие десорбции As $\theta_{L}=\theta_{0}-\theta+t / t_{\mathrm{ML}}$, где $t_{\mathrm{ML}}$ - время осаждения одного монослоя GaAs из пара.

На рис. 2 представлены зависимости приведенной свободной энергии образования островка $f+x \Delta \mu_{V S}$ как функции $\theta$ при различных значениях $x$ для ННК GaAs радиуса $20 \mathrm{~nm}$ при температуре $610^{\circ} \mathrm{C}$ и низком значении поверхностной энергии $\gamma_{0}=0.037 \mathrm{~J} / \mathrm{m}^{2}$. Значения $\theta_{e q}=0.34$ и $\theta_{0}=0.7$ дают размер остановки $\theta_{s}=0.36$ при нулевой накачке. По мере поступления атомов As из пара в каплю минимум свободной энергии, соответствующий текущему размеру островка, сдвигается вправо. Во всех случаях размер островка хорошо описывается объемной частью свободной энергии, показанной штриховыми линиями. Влияние поверхностной энергии островка на размер зародыша пренебрежимо мало. Иная картина наблюдается при высоком значении поверхностной энергии $\gamma_{0}=0.25 \mathrm{~J} / \mathrm{m}^{2}$. Свободная энергия образования зародыша, приведенная на рис. 3, имеет минимум, соответствующий размеру остановки, только при малых $x$, а затем минимум пропадает. Следовательно,

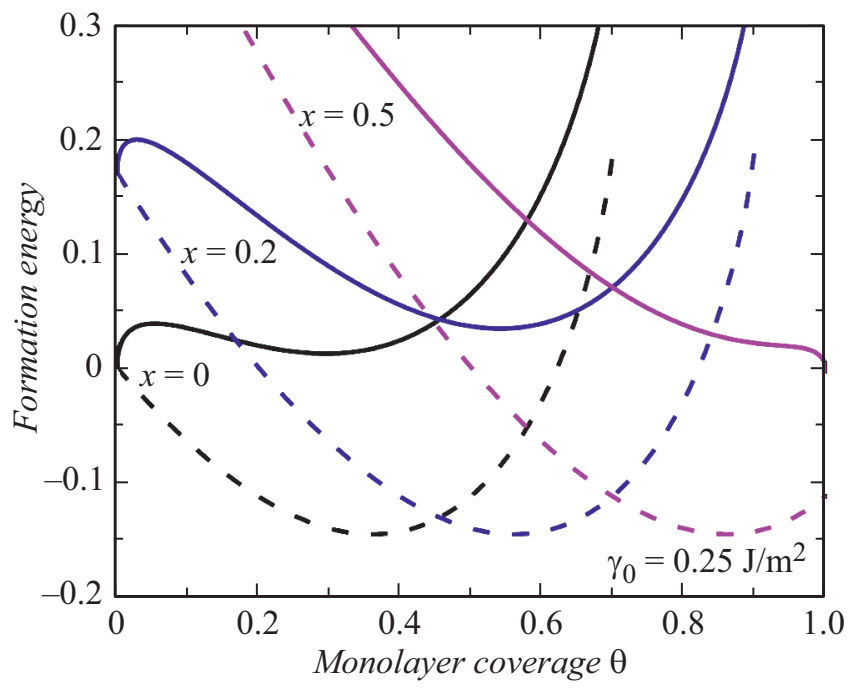

Рис. 3. То же, что на рис. 2 , при высоком значении поверхностной энергии $\gamma_{0}=0.25 \mathrm{~J} / \mathrm{m}^{2}$, соответствующем $\alpha=0.1056$. Размер остановки наблюдается при малых $x$ и исчезает при $x=0.5$ в результате уменьшения эффективной поверхностной энергии островка. Это соответствует быстрому росту островка из равновесной жидкости [17]. Объемная свободная энергия дает неправильную картину роста при больших $x$. 
по мере медленного роста островка за счет накачки из пара в какой-то момент островок вновь ускоряется и заполняет всю грань. Этот эффект связан с уменьшением поверхностной энергии островка [17]. В данном случае поверхностная энергия оказывает существенное влияние на характер роста.

В заключение отметим, что в работе приведен вывод свободной энергии образования зародыша при росте III-V ННК. Рассмотрение автокаталитического роста легко обобщается на случай капель $\mathrm{Au}$, что требует лишь переопределения равновесной концентрации As, которая будет зависеть от концентрации Ga.

\section{Финансирование работы}

Работа выполнена при поддержке Российского фонда фундаментальных исследований (гранты № 18-02-40006, 19-52-53031, 20-52-16301 и 20-02-00351).

\section{Конфликт интересов}

Авторы заявляют, что у них нет конфликта интересов.

\section{Список литературы}

[1] Zhang A., Zheng G., Lieber C.M. Nanowires: building blocks for nanoscience and nanotechnology. Springer, 2016. $327 \mathrm{p}$.

[2] Dubrovskii V.G. Theory of VLS growth of compound semiconductors // Semiconductors and Semimetals / Eds A. Fontcuberta i Morral, S.A. Dayeh, C. Jagadish. Burlington: Academic Press, 2015. V. 93. P. 1-78.

[3] Dimakis E., Jahn U., Ramsteiner M., Tahraoui A., Grandal J., Kong X., Marquardt O., Trampert A., Riechert H., Geelhaar L. // Nano Lett. 2014. V. 14. P. 2604-2609.

[4] Cirlin G.E., Bouravleuv A.D., Soshnikov I.P., Samsonenko Yu.B., Dubrovskii V.G., Arakcheeva E.M., Tanklevskaya E.M., Werner P. // Nanoscale Res. Lett. 2010. V. 5. P. 360.

[5] Wagner R.S., Ellis W.C. // Appl. Phys. Lett. 1964. V. 4. P. 8990.

[6] Dubrovskii V.G., Soshnikov I.P., Sibirev N.V., Cirlin G.E., Ustinov V.M. // J. Cryst. Growth. 2006. V. 289. P. 31-36.

[7] Matteini F., Dubrovskii V.G., Rüffer D., Tütüncüoğlu G., Fontana Y., Fontcuberta i Morral A. // Nanotechnology. 2015. V. 26. P. 105603.

[8] Cirlin G.E., Dubrovskii V.G., Petrov V.N., Polyakov N.K., Korneeva N.P., Demidov V.N., Golubok A.O., Masalov S.A., Kurochkin D.V., Gorbenko O.M., Komyak N.I., Ustinov V.M., Egorov A.Yu., Kovsh A.R., Maximov M.V., Tsatusul'nikov A.F., Volovik B.V., Zhukov A.E., Kop'ev P.S., Alferov Zh.I., Ledentsov N.N., Grundmann M., Bimberg D. // Semicond. Sci. Technol. 1998. V. 13. P. 1262-1265.

[9] Glas F. // Phys. Rev. B. 2006. V. 74. P. 121302(R).

[10] Ng K.W., Ko W.S., Tran T.T.D., Chen R., Nazarenko M.V., Lu F., Dubrovskii V.G., Kamp M., Forchel A., ChangHasnain C.J. // ACS Nano. 2013. V. 7. P. 100-107.

[11] Wen C.-Y., Tersoff J., Hillerich K., Reuter M.C., Park J.H., Kodambaka S., Stach E.A., Ross F.M. // Phys. Rev. Lett. 2011. V. 107. P. 025503.
[12] Harmand J.C., Patriarche G., Glas F., Panciera F., Florea I., Maurice J.-L., Travers L., Ollivier Y. // Phys. Rev. Lett. 2018. V. 121. P. 166101.

[13] Panciera F., Baraissov Z., Patriarche G., Dubrovskii V.G., Glas F., Travers L., Mirsaidov U., Harmand J.C. // Nano Lett. 2020. V. 20. P. 1669-1675.

[14] Reguera D., Bowles R.K., Djikaev Y., Reiss H.J. // J. Chem. Phys. 2003. V. 118. P. 340-353.

[15] Schmelzer J.W.P., Abyzov A.S. // J. Chem. Phys. 2011. V. 134. P. 054511.

[16] Dubrovskii V.G. // Cryst. Growth Design. 2017. V. 17. P. 2589-2593.

[17] Glas F., Dubrovskii V.G. // Phys. Rev. Mater. In press.

[18] Дубровский В.Г. // Письма в ЖТФ. 2020. Т. 46. В. 8. С. 3-6.

[19] Dubrovskii V.G., Hijazi H. // Nanomaterials. 2020. V. 10. P. 833.

[20] Dubrovskii V.G., Grecenkov J. // Cryst. Growth Design. 2015. V. 15. P. $340-347$. 